Management options

\title{
Anticoagulants for venous thrombosis
}

\author{
CH Toh
}

\begin{abstract}
Summary
The anticoagulant agents heparin and warfarin were introduced before the era of randomised clinical trials. As a result, the indications, dosages and monitoring techniques of these drugs have undergone re-evaluation in multiple clinical trials in the past years. Low molecular weight heparin has been developed, which has led to new approaches in anticoagulant management. Current levels of laboratory, pharmacology and clinical knowledge in the treatment of venous thromboembolism are discussed.
\end{abstract}

Keywords: anticoagulation, venous thromboembolism, low molecular weight heparin

Department of Haematology,

Royal Liverpool University Hospital, Prescot Street, Liverpool L7 8XP, UK $\mathrm{CH}$ Toh

Accepted 27 June 1996
The standard anticoagulant therapy for venous thromboembolism (deep vein thrombosis and/or pulmonary embolism) is a combination of parenteral heparin and oral warfarin. Anticoagulants (heparin in the 1930s and dicoumarol in the 1940s) came into use with meagre clinical data for their therapeutic effectiveness but with theoretical support. ${ }^{1}$ Since that time, the development of accurate objective tests to detect venous thromboembolism and advances in clinical trial methodology have led to substantial advancement in medical knowledge in this area. The practice of anticoagulant therapy can now be viewed as a model for evidence-based medicine.

\section{Heparin}

\section{STANDARD UNFRACTIONATED HEPARIN}

\section{Mechanism}

The anticoagulant activity of unfractionated heparin depends upon its unique pentasaccharide sequence that binds to antithrombin III, an endogenous anticoagulant protein. This facilitates and potentiates the inhibition of thrombin, a pivotal protein in thrombogenesis. ${ }^{2}$

\section{Limitations}

The highly negative charged nature of heparin renders it susceptible to binding by other plasma proteins, in particular platelet factor 4 and von Willebrand factor. This and other neutralising effects may explain why there is considerable inter-individual variability in the anticoagulant response to a fixed dose of heparin. In extreme circumstances, heparin resistance is encountered, often in the acutely unwell patient where high circulating levels of acute phase reactants are present.

\section{Monitoring}

The laboratory test to monitor heparin therapy is the activated partial thromboplastin time (APTT). Rigorously designed clinical trials have led to firm recommendations about the appropriate therapeutic range for the APTT. Failure to exceed the lower limits (APTT ratio of 1.5) is associated with an unacceptably high risk of recurrent venous thromboembolism. ${ }^{3}$ In contrast, no association exists between supratherapeutic APTT responses (an APTT ratio of 2.5 or more) and the risk of bleeding. ${ }^{4}$ Variability in the APTT response to different heparin blood levels supports the need for an aggressive approach to heparin therapy to ensure that all patients achieve adequate therapy early in the course of their treatment.

\section{LOW MOLECULAR WEIGHT HEPARIN}

\section{Mechanism}

The low molecular weight fractions of standard heparin with mean molecular weight ranges of 4000-5000 daltons (the molecular weight for unfractionated heparin is $12000-16000$ daltons) preferentially neutralises activated factor $\mathrm{X}$ (FXa), a key factor in the promotion of thrombin generation. ${ }^{5}$

\section{Advantages}

Unlike standard unfractionated heparin, low molecular weight heparin (LMWH) is not neutralised by platelet factor 4 . In studies on healthy volunteers, the bioavailability of LMWH after a single subcutaneous injection was approximately $90 \%$ of an equivalent intravenous dose. This excellent bioavailability of LMWH along with the longer half-life of its anticoagulant activity (as measured by anti-FXa activity) compared with unfractionated heparin, suggested that an effective regime for the initial treatment of venous thromboembolism using a once daily subcutaneous injection of LMWH might 


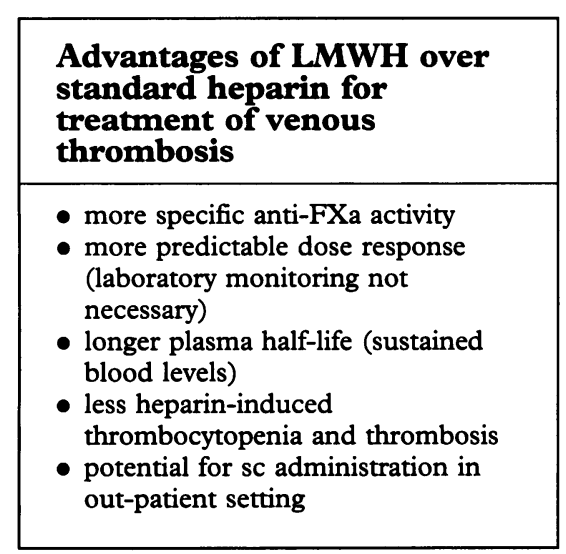

be possible. ${ }^{6}$ This could be given by a fixed dose (in terms of FXa units $/ \mathrm{kg}$ ) with no laboratory monitoring.

LMWH VS STANDARD UNFRACTIONATED HEPARIN

Although there is substantial literature on the clinical use of LMWH for the initial treatment of patients with proximal venous thrombosis, evidence-based medicine can only be drawn from large, blinded and randomised trials which have stringent design criteria and the power of definite results at the end.

\section{In-patient studies}

To date, five such trials fulfil the above criteria and compare LMWH subcutaneously, once or twice daily without monitoring, with intravenous standard unfractionated heparin used with stringent APTT monitoring to ensure therapeutic ranges in the treatment of deep vein thrombosis. ${ }^{7}$ In terms of outcome measures of recurrent venous thromboembolism and death, metaanalysis of these five trials has shown the superiority of LMWH over unfractionated heparin in a statistically significant manner. Meta-analysis of 10 studies in terms of safety (episodes of major bleeding) has also highlighted that LMWH is statistically significantly superior to standard heparin in this respect.

\section{Out-patient studies}

In view of the above results and the favourable pharmacology of LMWH, there is the potential for its use in an out-patient setting. Two large independent trials have now addressed this issue and shown that LMWH used at home is as effective and safe as in-patient continuous intravenous heparin with stringent APTT monitoring for the treatment of proximal vein thrombosis. ${ }^{8,9}$

\section{Warfarin}

\section{MECHANISM}

Warfarin produces its anticoagulant effect by interfering with vitamin $\mathrm{K}$ dependent coagulant factors II, VII, IX and X. In view of the long half-life of the vitamin-K-dependent coagulant factors, warfarin therapy must overlap with heparin therapy for three to five days with discontinuation of heparin once a therapeutic International Normalised Ratio (INR) range is reached. A number of studies have shown that the traditional approach of a 10-day course of heparin therapy with warfarin starting on day five or six is unnecessary. ${ }^{10,11}$ Starting warfarin simultaneously or soon after heparin is initiated, produces no short or long-term differences in outcome and leads to shorter hospital stays, thus being a more cost-effective approach to treatment. Such treatment may even be safer in terms of the potential lethal complication of heparin-induced thrombocytopenia and thrombosis syndrome. ${ }^{12}$

\section{LIMITATIONS}

Oral anticoagulants may be involved in serious, life-threatening adverse reactions in the presence of other drugs. The mechanisms by which the pharmacokinetic and pharmacodynamic responses of warfarin are affected include induction or inhibition of hepatic enzyme activity, alteration of vitamin $\mathrm{K}$ absorption, and displacement from plasma protein-binding sites.

\section{MONITORING}

Differences in the sensitivity of thromboplastin reagents which have led to inconsistencies in prothrombin time (PT) ratios, non-comparability of results from different laboratories or even within the same laboratory as reagents change, have led to the uniform acceptance of the INR as a means of equilibrating PT ratios to effect safer and better therapies. The INR converts the PT ratio to an internationally accepted norm based on the sensitivity of the thromboplastin used in the assay. There is evidence that the therapeutic range as defined by an INR of 2-3 is effective in patients at risk of recurrent venous thromboembolism. ${ }^{13}$ In patients with the antiphospholipid antibody syndrome, a somewhat higher therapeutic range is recommended (INR of 3-4). ${ }^{14}$ Such levels should also be aimed at for in-patients with mechanical heart valves. ${ }^{15}$

\section{DURATION OF TREATMENT}

Two large randomised trials have tried to determine the optimal duration of treatment with oral anticoagulants. ${ }^{16,17}$ There is insufficient evidence to support life-long treatment for all patients with idiopathic venous thrombosis. On the basis of the published observations, it would be reasonable to pursue anticoagulant therapy for six weeks in patients with reversible risk factors and 
to continue anticoagulation for up to six months in patients with idiopathic venous thrombosis. Long-term anticoagulant therapy should be considered for patients who have recurrent episodes of idiopathic deep vein thrombosis and those with inherited thrombophilia who have had one or more unprovoked episodes of major venous thromboembolism.

\section{Conclusions}

The major impact on anticoagulant practice in terms of healthcare delivery at the current time is the potential for a shift from in-patient to out-patient management. The use of LMWH as the initial therapy for deep vein thrombosis in the out-patient setting has been shown to be effective and safe. Although there is literature on its cost-effectiveness, the major benefit in real terms will be in the relieving of acute bed shortages and reducing the burden on acutely stretched junior doctors. Such a change in practice requires a flexibility of approach and co-ordination of care, possibly by a core anticoagulant team to ensure appropriate warfarin adjustments, monitoring, and timing of heparin termination. The time-honoured tradition of Anticoagulant Clinics purely as places for warfarin monitoring and dosing needs to be rethought to meet the challenge of providing a more comprehensive anticoagulant service; one which could now encompass the acute management of patients with venous thromboembolism.

1 Wright IS, Prandoni A. The dicoumarin 3,3' methylene-bis-(4-hydroxycoumarin): its pharmacologic and therapeutic action in man. $\mathscr{f} A M A$ 1942; 120: $1015-21$. 2 Rosenberg RD, Damus PS. The purification and mechanism of action of human antithrombin-
heparin co-factors. $₹$ Biol Chem 1973; 248: heparin co-

3 Hull RD, Raskob GE, Hirsh J, et al. Continuous intravenous heparin compared with intermittent subcutaneous heparin in the initial treatment of proximal-vein thrombosis. $N$ Engl f Med 1986; 315: $1109-14$

4 Hull RD, Raskob GE, Pineo GF, et al. Subcutaneous low molecular weight heparin compared with continuous intravenous heparin in the treatment of proximal-vein thrombosis. $N$ the treatment of proximal-vein

5 Verstraete M. Pharmacotherapeutic aspects of Verstraete $M$. Pharmacotherapeutic aspects of
unfractionated and low molecular weight heparin. Drugs 1990; 40: 498-530.

6 Matzsch T, Bergqvist D, Hedner U. Effects of enzymatically depolymerised heparin as compared with conventional heparin in healthy volunteers. Thromb Haemost 1987; 57: 97-101.
7 Lensing AWA, Prins MH, Davidson BL, Hirsh J. Treatment of deep vein thrombosis with low molecular weight heparins - a meta-analysis. Arch Intern Med 1995; 155: 601-7.

8 Levine M, Gent M, Hirsh J, et al. A comparison Levine M, Gent M, Hirsh J, et al. A comparison
of low molecular weight heparin administered of low molecular weight heparin administered
primarily at home with unfractionated heparin primarily at home with unfractionated heparin
administered in the hospital for proximal deep vein thrombosis. N Engl f Med 1996; 334: 67781.

9 Koopman MMW, Prandoni P, Piovella F, et al. Treatment of venous thrombosis with intravenous unfractionated heparin administered in the hospital as compared with subcutaneous low mospital as compared N Engl ₹ Med 1996; 334: 682-7.

10 Gallus A, Jackaman J, Tillett J, et al. Safety and efficacy of warfarin started early after submassive venous thrombosis or pulmonary embolism. venous thrombosis or
Lancet 1986; 2: 1293-6.

11 Hull RD, Raskob GE, Rosenbloom D, et al. Heparin for 5 days as compared with 10 days in initial treatment of proximal venous thrombosis. $N$ Engl f Med 1990; 322: 1260-4.
12 Mohiuddin SM, Hilleman DE, Destache CJ, et al. Efficacy and safety of early versus late initiation of warfarin during heparin therapy in initiation of warfarin during heparin therapy in 729-32.

13 British Society for Haematology. Guidelines on oral anticoagulation: second edition. $f$ Clin Pathol 1990; 43: $177-83$.

14 Khamashta MA, Cuadrado MJ, Mujic FM, et al. The management of thrombosis in the antiphospholipid-antibody syndrome. $N \mathrm{Engl} f \mathrm{Med}$ 1995; 332: $993-7$.

15 Busuttil WJ, Fabri BM. The management of anticoagulation in patients with prosthetic heart valves undergoing non-cardiac operations. Postgrad Med f 1995; 71: 390-2.

16 British Thoracic Society Research Committee. Optimal duration of anticoagulation for deep Optimal duration of anticoagulation for deep
vein thrombosis and pulmonary embolism. vein thrombosis and pal

17 Schulman S, Rhedin AS, Lindmarker P, et al. A comparison of 6 weeks with 6 months of oral anticoagulant therapy after a first episode of venous thromboembolism. $N$ Engl f Med 1990; 332: $1661-5$. 